\title{
Variación espacial y temporal de los organismos de un intermareal rocoso: Bahía Panamá, Pacífico Norte, Costa Rica
}

\author{
Jeffrey A. Sibaja-Cordero ${ }^{1,2} \&$ Kimberly García-Méndez ${ }^{2}$ \\ 1. Centro de Investigación en Ciencias del Mar y Limnología (CIMAR), Universidad de Costa Rica, San Pedro, 11501- \\ 2060 San José, Costa Rica; jeffrey.sibaja@ucr.ac.cr \\ 2. Escuela de Biología, Universidad de Costa Rica, San Pedro, 11501-2060 San José, Costa Rica.
}

\author{
Recibido 22-III-2014. Corregido 03-VI-2014. Aceptado 27-IX-2014.
}

\begin{abstract}
Spatial and temporal variation of rocky intertidal organisms: Bahía Panamá, North Pacific, Costa Rica. The intertidal rocky shore of Bahía Panama, within Bahía Culebra in the North Pacific coast of Costa Rica, was sampled to study the biodiversity and the spatial patterns (three sites with differences in wave exposure, and five strata, from low to high tide levels) and temporal variation (a single date in 2007 and 2013) in different microhabitats (over and under rock surface, and crevices). The epibenthic organisms were sampled with quadrats of $25 \times 25 \mathrm{~cm}$ in each stratum, and cryptic fauna was accounted by both visual census and manual collecting. The general pattern of vertical zonation of organisms at each site showed an increase in variety toward the infralittoral level, as well as in the composition of assemblages or partially in identity of taxa in each stratum. The sheltered section of the rocky coast, with shallow sand bottom $(<1 \mathrm{~m})$, low wave action and greater sedimentation rate, had lower diversity, cover and abundance that increased towards the wave-exposed section, with more abrupt slope in the edge of rocky platform, lower turbidity, and deeper sand bottom (2-3 m). The horizontal change was more evident in epibenthic organisms, followed by the under rock surface inhabitants and finally in crevices. For the epibenthic organisms, a temporal change in assemblages (cover and abundance) was found between the 2007 and 2013, at each site and level (possibly by seasonal upwelling influence), but the spatial structure (vertical and horizontal) of the rocky shore seems the main cause of change in the composition of species. The spatial pattern of change in the biota (sheltered to exposed gradient) could be expected in similar shores of the Central American Pacific. Finally, the 157 species found (16 autotrophic taxa, 22 fishes, and 119 invertebrates) indicate the high biodiversity of this habitat in the tropics, and the importance of their conservation. Rev. Biol. Trop. 62 (Suppl. 4): 85-97. Epub 2014 Diciembre 01.
\end{abstract}

Key words: assemblage variability, invertebrates, microhabitats, marine biodiversity.

Las zonas rocosas tropicales de Centro América han sido tradicionalmente catalogadas como hábitats de poca diversidad, a partir de trabajos realizados en Costa Rica y Panamá, donde el objeto de estudio ha sido básicamente la epifauna del substrato rocoso. Por ejemplo, Paine (1966) indica una simplicidad de las cadenas tróficas en el trópico; Menge \& Lubchenco (1981) resaltan la baja diversidad faunística y la presencia de esta solo en grietas en Panamá; Sutherland \& Ortega (1986) y Ortega (1987) indican, asimismo, la dominancia de unas pocas especies de gasterópodos en el Pacífico Central de Costa Rica. Las zonas rocosas costarricenses, por ejemplo, cuentan con ciertos organismos sésiles de la epifauna que dominan o monopolizan el substrato, entre ellos se encuentran los cirripedios Chthamalus panamensis Pilsbry, 1916 y Tetraclita stalactifera (Lamarck, 1818), los bivalvos Saccostrea palmula (Carpenter, 1857), Ostrea virescens Angas, 1868 y Brachidontes spp., representados prácticamente a lo largo del litoral Pacífico (Villalobos, 1980a; Villalobos 1980b; Ortega, 1987). Entre la fauna móvil destacan los litorínidos Echinolittorina aspera (Philippi, 1846) 
y Echinolittorina modesta (Philippi, 1846), los caracoles Nerita scabricosta Lamarck, 1822 y Nerita funiculata Menke, 1851, y los murícidos Acanthais brevidentata (Wood, 1828), que pueden tener altas densidades (Bakus, 1968; Spight, 1977). En las zonas rocosas se incluyen organismos menos visibles que viven entre las grietas, pozas de marea, debajo de las rocas o en las algas marinas, todos ellos microhábitats donde puede darse una vasta riqueza.

Por otro lado, trabajos como los de Fischer (1981), Sibaja-Cordero \& Vargas-Zamora (2006) y Sibaja-Cordero \& Cortés (2008), incluyen aspectos de cobertura y densidades de algas e invertebrados, tomando en cuenta las especies ocasionales y poco abundantes. Pese a ello, estos trabajos pocas veces mencionan más de 20 especies. Sin embargo, los trabajos más enfocados hacia la determinación de la riqueza de especies no han abarcado la totalidad de la comunidad, si bien han demostrado que la diversidad de los grupos estudiados suma más especies que las reportadas en los estudios meramente ecológicos, como Villalobos (1980b) con algas, Spight (1977) o Miller (1974) y Willis \& Cortés (2001), con moluscos. Pocos trabajos hacen mención a organismos que viven en grietas aparte de los de Fischer (1981), Pepe (1985) y Dean (2001).

La mayoría de la fauna que vive bajo las rocas y en grietas ha sido poco estudiada en Costa Rica, representando un importante vacío de información, y que no se ha tomado en cuenta en la toma de decisiones de la gestión de la línea costera. Además, el estudio de la variación espacial (vertical-horizontal) y temporal, incluyendo diversos microhábitats de la zona intermareal no se ha realizado aún para Costa Rica. Es importante que en futuros estudios de las zonas rocosas en zonas tropicales se haga una evaluación que incluya todos los componentes de su biodiversidad. En este sentido, este trabajo busca incluir algunos de estos grupos para describir el patrón de variación en la distribución vertical y horizontal de organismos en los microhábitats de la zona intermareal de Bahía Panamá, desde la sección más protegida a otra con mayor influencia de corrientes y oleaje.

\section{MATERIALES Y MÉTODOS}

Se visitó la zona rocosa intermareal de Bahía Panamá, ubicada en Bahía Culebra, Guanacaste, costa Pacífica de Costa Rica (Fig. 1). Dicha localidad se visitó el 13 de diciembre de 2007, durante la marea baja; para realizar el estudio de la epifauna del intermareal. Otra visita se realizó el 10 de agosto de 2013, para obtener una lista de la epifauna y los organismos crípticos (habitantes de grietas y bajo las rocas), presentes en el intermareal. En ambas visitas, se dividió la zona rocosa en tres zonas, desde la zona A ubicada en la parte interna de Bahía Panamá, hasta la zona $\mathrm{C}$ en la zona más expuesta de esta bahía (Fig. 1). La zona intermareal se dividió en sentido vertical en cinco estratos, desde el nivel de marea baja de las mareas vivas (estrato 1) hasta la zona superior del intermareal (supralitoral, estrato 5) y anterior a los líquenes y vegetación terrestre.

\section{Cobertura y abundancia del interma-}

real: En 2007, en cada estrato y dentro de cada sitio, se establecieron 10 cuadrículas de 25 por $25 \mathrm{~cm}$ y a cada una se le tomó una fotografía. En 2013 se tomo una fotografía de una cuadrícula de cada estrato en cada sitio para comparar con los datos de 2007. Las imágenes se analizaron en el programa UTHSCSA Image Tool para estimar la cobertura de los organismos sésiles y la abundancia de la fauna móvil. Se empleó los filtros del programa Adobe Photoshop, para resaltar tonalidades de la especie de interés y subsecuentemente en Image Tool se realizó la estimación de la cobertura mediante análisis en escala de grises o por substracción de la imagen editada con la original según Sibaja-Cordero (2008). La abundancia de las especies móviles en las cuadrículas se determinó con el comando "point" (Sibaja-Cordero, 2008). Los moluscos se identificaron con Keen (1971) y los cirripedios con Laguna (1985). 

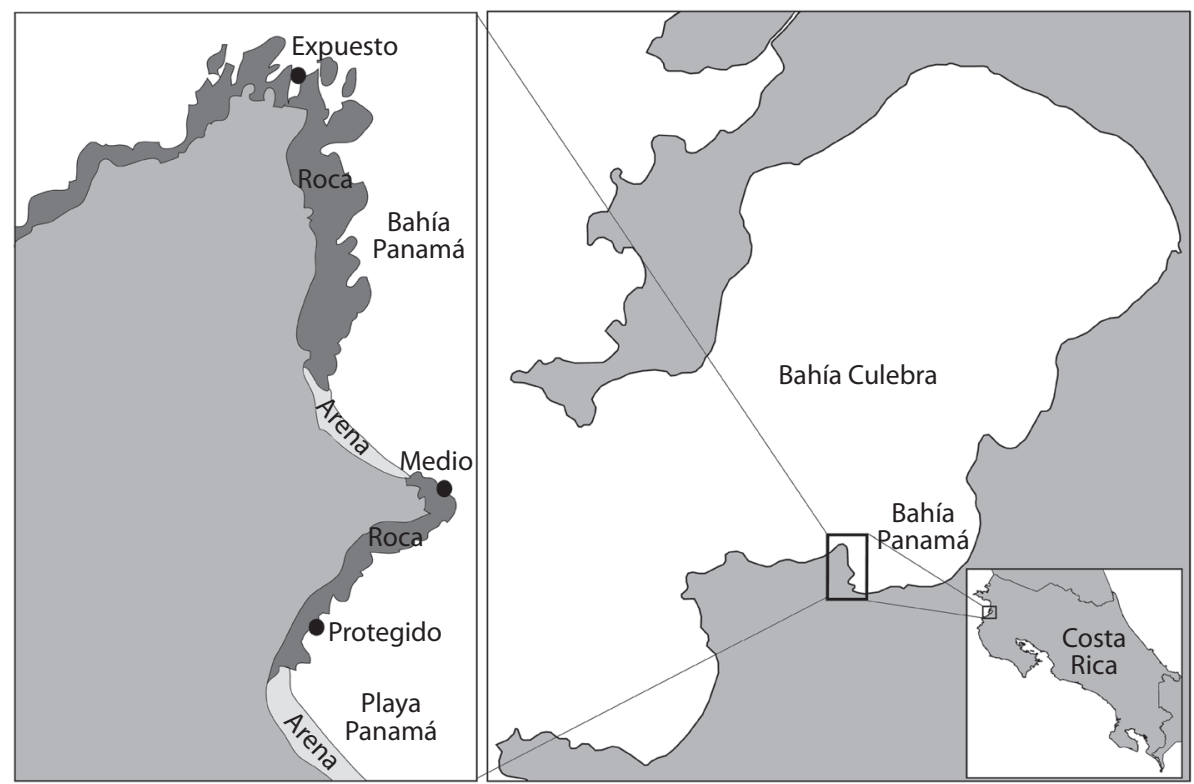

Fig. 1. Ubicación de los puntos de muestreo en la Bahía Panamá, Pacífico Norte de Costa Rica.

Epifauna y fauna críptica: En 2013, se recorrió el intermareal y se censaron todas las especies de epifauna presentes. Además, con la ayuda de mazo y cincel se procedió a remover la fauna presente en grietas desde los niveles supralitoral a infralitoral. También, a lo largo del intermareal se buscó la fauna presente bajo las rocas. Adicionalmente, se buscó empleando careta de buceo, la fauna presente en las algas de la zona, tanto en la roca expuesta como en los canales, pozas intermareales y el infralitoral. Todos los organismos avistados y recolectados se clasificaron hasta la categoría taxonómica más específica posible, ya fuese especie o bien morfotipo.

Peces y algas: Se hizo una lista, mediante buceo a pulmón, de los peces que visitaban el infralitoral así como de los principales grupos o formas algales presentes. Las especies de peces se identificaron con la literatura disponible para la región (Humann \& DeLoach, 2004).

Análisis de datos: Para la zona intermareal se generó una matriz de datos (cuadrículas en las filas y especies en las columnas) de cobertura y abundancia de la epibiota. Se transformó con raíz cuarta los porcentajes de cobertura (especies sésiles) y con log $(\mathrm{x}+1)$ la abundancia (especies móviles). Las columnas se estandarizaron (promedio $=0$ y desviación estándar $=1$ ). Se realizó un Análisis de Componentes Principales (PCA) a partir de los datos transformados y estandarizados en el paquete PAST (Hammer, Harper \& Ryan, 2001; Quinn \& Keough, 2003), para mostrar la similitud de las cuadrículas de las diferentes zonas y estratos del intermareal rocoso de Bahía Panamá. En el PCA, se representó con vectores para las especies la tasa y la dirección en que se incrementó la abundancia o cobertura de las diferentes especies (Quinn \& Keough, 2003). Se generó una matriz basada en la distancia euclidiana entre las cuadrículas y se analizó con un ANOSIM (R) de dos vías en PAST (Hammer, Harper \& Ryan, 2001; Clarke \& Warwick, 1994), para determinar diferencias en la composición de organismos entre las zonas (A, B y C) y los estratos del intermareal (1 al 5). El valor de $\mathrm{R}$ indica el grado de diferencia entre niveles de los distintos factores, 
obteniéndose $\mathrm{R}=1$ en caso de que los grupos fuesen completamente diferentes uno del otro.

Además, se realizó un PCA incorporando las cuadrículas del 2013 a los datos del 2007, que se presentan como centroides por sitio-estrato. Se realizó un PERMANOVA de dos vías para determinar si hubo un cambio espacio-temporal, así como interacción entre dichos factores, en la composición de las especies del intermareal respecto a las zonas y los años 2007 y 2013 (Hammer, Harper \& Ryan, 2001; Quinn \& Keough, 2003).

Se realizó una lista de todas las especies presentes por zona a partir de los datos obtenidos en la visita de 2013. Se contabilizaron las especies según el grupo taxonómico superior o grupo funcional. Las posibles diferencias en función del número de especies se contrastó por medio de una prueba chi cuadrado. Además, se presenta la similitud entre la zona protegida, media y expuesta por medio del índice de Sorensen (Dice), junto con el número de especies que solo se encontraron en cada zona (exclusivas) y el de especies compartidas entre estas (comunes). El cambio en la composición se contrastó con una prueba $\mathrm{Z}$ de seriación en PAST, respecto al grado de exposición al oleaje. Además, se contabilizó la diversidad puntual por zona y microhábitat: sobre o bajo las rocas y en las grietas, y de esta manera, se calculó el índice de Sorensen para comprobar si algún grupo de fauna, según microhábitat, presentaba o no una mayor o menor similitud ante la exposición al oleaje.

\section{RESULTADOS}

\section{Variación vertical de la comunidad:}

Abundancia y cobertura del intermareal. Por medio del Análisis de Componentes Principales (Fig. 2) se encontró que el estrato 1 (infralitoral) de los sitios en la zona rocosa de Bahía Panamá, se segregó de los demás estratos mostrando una gran dispersión. En los estratos 2 y 3 (litoral bajo y medio) se dio una mezcla de

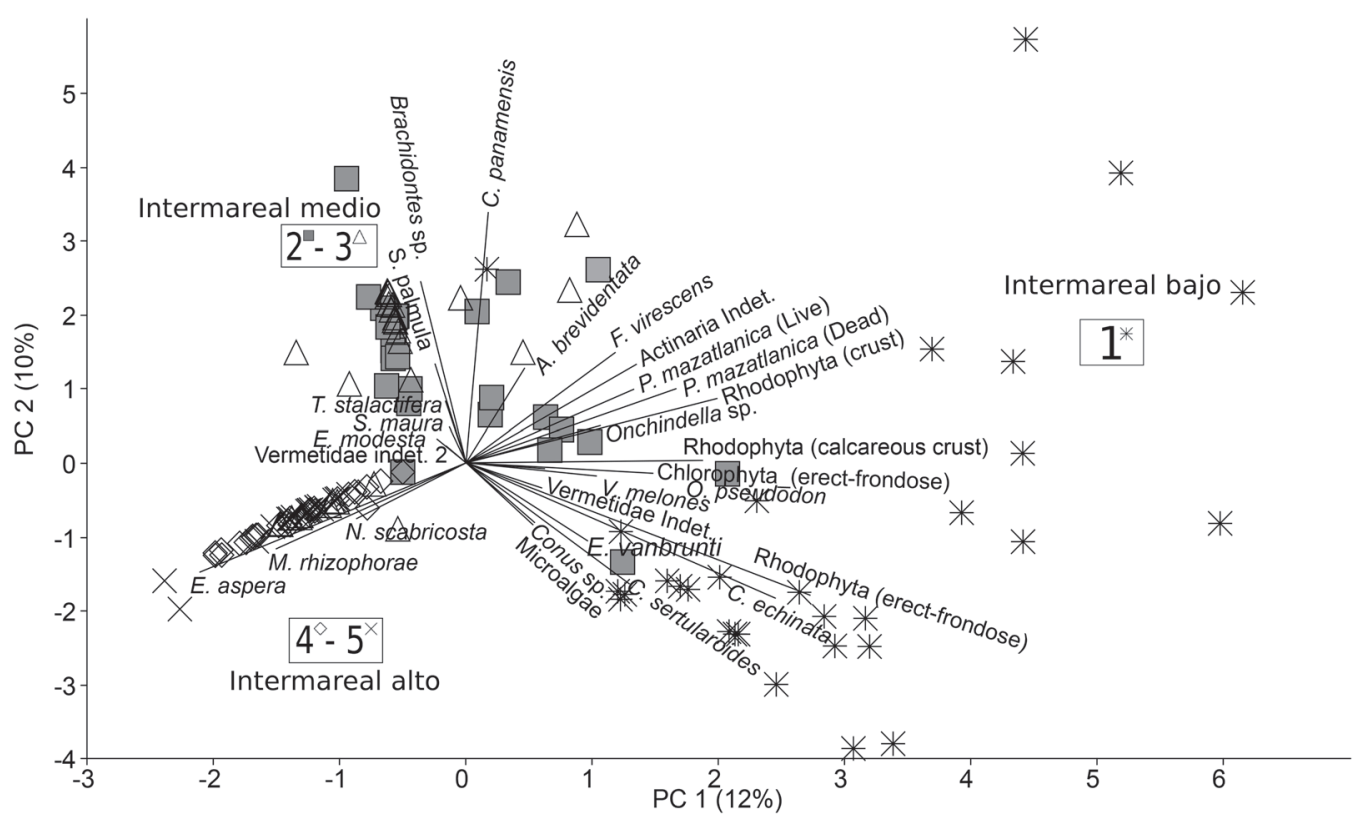

Fig. 2. Análisis de Componentes Principales de la abundancia o cobertura por estrato de los organismos de la zona rocosa de Bahía Panamá, Costa Rica. Números: estratos (1 es el más cercano al nivel de marea baja y 5 el más alto del supralitoral). Líneas: vectores de las especies que indican la dirección y tasa hacia donde se incrementó la abundancia o cobertura. Diciembre 2007. 
las diferentes cuadrículas debido a la similitud de su biota. Los estratos 4 y 5 (litoral superior y supralitoral) también se agruparon; el nivel superior mostró una menor dispersión en la composición de sus biotas. La composición de los organismos varió significativamente desde el nivel de marea baja hacia la zona superior del intermareal; con un grado de diferencia del $40 \%$ entre estratos $(R=0.40, p<0.001)$.

Distribución vertical de las especies. Las especies en el intermareal rocoso mostraron una típica distribución de zonación vertical (PCA en Fig. 2), junto con las observaciones hechas en el campo, con algas, bivalvos y los gasterópodos Vasula melones (Duclos, 1832) y Opeatostoma pseudodon (Burrow, 1815) en el infralitoral y litoral bajo. El alga Caulerpa sertularoides (S. G. Gmelin) M. A. Howe, 1905, cubrió casi completamente gran parte de la franja infralitoral y algunas de las pozas intermareales en los niveles bajos. Destacó en este nivel la presencia de la ostra perlera Pinctada mazatlanica (Hanley, 1856) y del pulmonado Onchindellla sp., apareciendo muchas veces incluso dentro de las grietas de las rocas. Otros moluscos comunes fueron las babosas marinas Dolabrifera dolabrifera (Rang, 1828) y Spurilla neapolitana (Delle Chiaje, 1841), principalmente en la pozas intermareales. El litoral medio comprendió poblaciones de los cirripedios $T$. stalactifera y $C$. panamensis, la lapa pulmonada Siphonaria maura Sowerby, 1835, los bivalvos $S$. palmula y Brachidontes sp., el gasterópodo depredador $A$. brevidentata y el gasterópodo herbívoro Fisurella virescens G. B. Sowerby, 1835. El litoral alto estuvo dominado por el burgado $N$. scabricosta, el caracol E. aspera y el cirripedio Microeuraphia rhizophorae (De Oliveira, 1940).

\section{Variación horizontal y temporal de la comunidad:}

Abundancia y cobertura del intermareal. Además, cierto grado de diferencia horizontal en la composición por cobertura y abundancia se dió entre las secciones del intermareal desde la parte interna de la Bahía Panamá hacia el sector externo (Fig. 3), que fue detectado con los análisis ANOSIM $(\mathrm{R}=0.26, \mathrm{p}<0.001)$ y PERMANOVA (Cuadro 1). Esta última prueba indica que el gradiente de cambio en composición desde zona protegida a expuesta se dio en el infralitoral en 2007 y 2013 de la misma manera, siendo más diferente el sitio protegido.

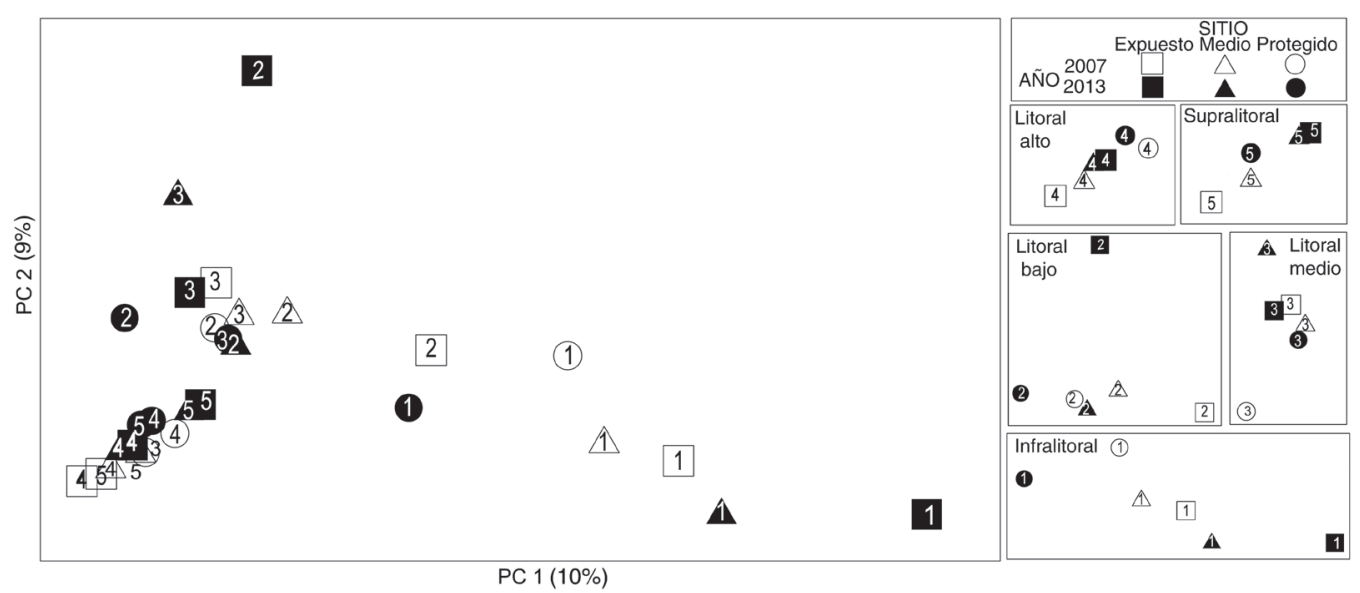

Fig. 3. Resumen del PCA, mostrando la ubicación de los centroides de los valores de cada estrato por localidad y año, basado en la disimilitud de la abundancia o cobertura de los organismos en la zona rocosa. Números: estratos (1 es el más cercano al nivel de marea baja y 5 el más alto del supralitoral). A la derecha, se presenta por separado cada estrato, para mostrar el efecto de año y la localidad según el PERMANOVA del Cuadro 6. Bahía Panamá, Costa Rica. 
CUADRO 1

Efecto de la localidad (expuesto, medio y protegido) y el año (2007 vrs 2013) sobre la disimilitud (Distancia euclidiana) de la biota de la zona rocosa de entre mareas, por medio de una prueba de PERMANOVA de dos vías. Bahía Panamá, Costa Rica

\begin{tabular}{lcccccccccc} 
& \multicolumn{1}{c}{} & \multicolumn{1}{c}{ L } & \multicolumn{1}{c}{ Estrato } \\
& Infralitoral & \multicolumn{2}{c}{ Litoral bajo } & \multicolumn{2}{c}{ Litoral medio } & \multicolumn{2}{c}{ Litoral alto } & \multicolumn{2}{c}{ Supralitoral } \\
Factor & $\mathrm{F}$ & $\mathrm{p}$ & $\mathrm{F}$ & $\mathrm{p}$ & $\mathrm{F}$ & $\mathrm{p}$ & $\mathrm{F}$ & $\mathrm{p}$ & $\mathrm{F}$ & $\mathrm{P}$ \\
Localidad & 1.27 & $\mathbf{0 . 0 2 3}$ & 2.14 & $\mathbf{0 . 0 2 4}$ & 3.55 & $\mathbf{0 . 0 0 5}$ & 5.74 & $<\mathbf{0 . 0 0 1}$ & 1.87 & $\mathbf{0 . 0 3 2}$ \\
Año & 0.82 & $\mathbf{0 . 0 0 4}$ & 1.11 & $\mathbf{0 . 0 0 3}$ & 3.55 & $<\mathbf{0 . 0 0 1}$ & 6.74 & $<\mathbf{0 . 0 0 1}$ & 0.93 & 0.066 \\
Interacción & -5.99 & 0.188 & -4.36 & 0.068 & -4.09 & $\mathbf{0 . 0 0 8}$ & -6.07 & 0.257 & -6.19 & 0.993 \\
\hline
\end{tabular}

En el litoral bajo (estrato dos) el sitio más diferente fue el expuesto (Fig. 3) en ambos años. En el litoral medio (estrato 3 ) se da un cambio en el patrón (interacción entre sitioaño en Cuadro 1), ya que en 2007 el sitio más diferente fue el protegido, mientras en 2013 lo fue el medio. El litoral alto (estrato 4) presenta solamente diferencia del sitio protegido del resto, en ambos años, mientras el supralitoral (estrato 5) presenta el sitio expuesto de 2007 diferente a los demás. Se halló diferencia en la composición de epibiota entre 2007 y 2013 (Cuadro 1), salvo para el supralitoral, y dicha diferencia se debe al número de especies en las cuadrículas, su cobertura y abundancia.

Por ejemplo, se encontró en 2013 respecto a 2007, en el infralitoral (estrato 1), un incremento de E. aspera, con algunos individuos en las zonas media y protegida; las algas verdes se incrementaron en la zona expuesta, pero se redujeron en la media y protegida y el alga incrustante Ralfsia sp. se incrementó en todo el infralitoral. Para otras 18 especies se detectó una reducción en cobertura o abundancia en 2013. En el litoral bajo (estrato 2), se encontró más $C$. sertularoides y otras algas verdes que en 2007 en la zona media y protegida; por el contrario, las rodófitos mostraron una menor cobertura. Once especies mostraron un reducción de sus poblaciones y tres invertebrados (E. aspera, E. vanbrunti y una nerita) tuvieron incrementos puntuales en su densidad en diferentes zonas dentro de este estrato. En el litoral medio (estrato 3), el bivalvo S. palmula y el alga incrustante Ralfsia sp. se incrementaron en todas las zonas; por el contrario, las litorinas, los cirripedios, las anémonas y el bivalvo Brachidontes sp. mostraron un descenso. En el litoral alto (estrato 4), para los cirripedios, $C$. panamensis se incrementó y $M$. rhizophorae se redujo, mientras que el bivalvo Brachidontes sp. y el alga Ralfsia sp. mostraron el patrón opuesto. La abundancia de litorina E. aspera aumentó su densidad en la zona media y la de E. modesta disminuyó en el interior de la bahía. El estrato supralitoral no presentó cambios significativos entre los años muestreados.

\section{Distribución horizontal de las especies} en la costa. En el muestreo realizado en 2013 se censaron 157 especies en la zona rocosa de Bahía Panamá. La riqueza fue mayor en la zona más expuesta y decreció hacia la zona protegida (Cuadro 2 y 3 ).

En total, se encontraron 45 especies de moluscos, la mayoría caracoles, 17 de poliquetos, 15 de equinodermos y 12 de crustáceos. Otros grupos contaron con menos de diez especies. Además, 22 especies de peces visitan la zona rocosa de Bahía Panamá y 14 tipos de algas se encuentran creciendo sobre la roca (Cuadro 2). La tendencia general de varios de estos grupos fue presentar mayor riqueza en la zona más expuesta y menor diversidad en la protegida (Cuadro 3), por lo que en general la distribución de la biota no fue azarosa $(\mathrm{z}=$ $-3.42, p<0.001)$ y siguió un gradiente desde el interior de las bahía hacia la zona con mayor oleaje y corrientes. La zona expuesta sumó más de cien especies mientras que la protegida menos de 50 (Cuadro 3). 


\section{CUADRO 2}

Organismos presentes en la costa rocosa de Bahía Panamá, Pacífico Norte. Agosto 2013. Para cada taxón se indica en que zona se halló: $*=$ Expuesto, ${ }^{\circ}=$ Medio y ${ }^{\mathrm{a}}=$ Protegido del oleaje. La riqueza de taxones se indica entre paréntesis

\begin{tabular}{ll}
\multicolumn{1}{c}{ Filo/Subfilo/Clase } & \multicolumn{1}{c}{ Taxones } \\
Cyanobacteria (2) & Lyngbya sp.*; Cyanobacteria indet. ${ }^{* 0}$. \\
Chlorophyta (4) & Caulerpa: $\left(\right.$ C. peltata J.V.Lamouroux, $1809^{* 0} ;$ C. racemosa $($ Forsskål) J.Agardh, \\
& $1873^{* 0} ;$ C. sertularioides (S.G.Gmelin) M.A.Howe, 1905*0a); Chlorophyta \\
& $(\text { erecta-frondosa })^{* 0 a}$.
\end{tabular}

Rhodophyta (7)

Ochrophyta (3)

Porifera (7)

Cnidaria/Anthozoa (8)

Cnidaria/Hydrozoa (3)

Platyhelminthes/Turbellaria (2)

Bryozoa (5)

Mollusca/Bivalvia (10)

Mollusca/Gastropoda (32)

Mollusca/Polyplacophora (3)

Sipuncula (3)

Annelida/Polychaeta (17)

Arthropoda/Crustacea/Malacostraca (7)
Amphiroa sp.*o; Peyssonelia sp.*; Rhodophyta taxones indet.: (calcárea articulada*; calcárea incrustante*oa; calcárea incrustante $\mathrm{A}^{*}$; erecta-frondosa*oa; filamentosa*o ${ }^{*}$.

Ralfsia sp.*aa; Dictyota: (D. humifusa Hörnig, Schnetter \& Coppejans, 1992*; D. stolonifera E.Y. Dawson, 1962*).

Porifera:(indet.*oa; indet. $\mathrm{A}^{*}$; indet. $\mathrm{B}^{\mathrm{o}}$; indet. $\mathrm{C}^{\mathrm{o}}$; indet. $\mathrm{D}^{\mathrm{a}}$; indet. $\mathrm{E}^{\mathrm{o}}$; indet. $\mathrm{F}^{\mathrm{o}}$ ).

Zoanthidea indet.*; Epizoanthus sp.*; Bunodosoma sp.*; Actinaria: (indet.*oa; indet. $\mathrm{A}^{*}$; indet. $\mathrm{B}^{\mathrm{o}}$ ); Leptogorgia alba ${ }^{* \mathrm{o}}$; Octocorallia indet. ${ }^{\mathrm{o}}$

Hydrozoa: (indet.*; indet. $\mathrm{A}^{\mathrm{o}}$; indet. $\mathrm{B}^{*}$ )

Polycladida: (indet. ${ }^{\circ}$; indet. $A^{*}$ ).

Bryozoa: (indet.*o; indet. $\mathrm{A}^{*}$; indet. $\mathrm{B}^{\mathrm{o}}$ ); Membranoporidae: (indet.*; indet. $\mathrm{A}^{\mathrm{o}}$ ).

Bivalvia indet. '; Chama echinata Broderip, 1835% Chama sp.*; Brachidontes sp. $^{* 0 a}$; Lithophaga sp. $^{\circ}$; Ostrea sp..$^{\text {a. }}$ Ostrea virescens Angas, $1868^{* 0 a}$; Ostreidae intet. ; Pinctada mazatlanica (Hanley, 1856) ${ }^{* 0}$; Pteridae indet..; Saccostrea palmula (Carpenter, 1857)*oa

Gastropoda : (indet.*; indet. A*); ?Acmeidae indet.*o; Acanthais brevidentata (Wood, 1828)*oa; Cerithidae indet.*; Conus nux Broderip, 1833º; Conus sp.*; Dolabrifera dolabrifera (Rang, 1828)*o; Doriopsilla janaina Er. Marcus \& Ev. Marcus, 1967*; Echinolittorina aspera (Philippi, 1846)*oa. Echinolittorina modesta (Philippi, 1846) ${ }^{\circ}$; Fissurella virescens G. B. Sowerby, $1835^{\text {a }}$; Fissurella sp.*; Fissurellidae indet.*; Hexaplex princeps (Broderip, 1833)*o; Lottia sp.*; Nerita funiculata Menke, 1851*0; Nerita scabricosta Lamarck, 1822*o; Nerita sp. ${ }^{\text {a }}$ N Neritidae indet.*; Onchidella sp.*oa; Opeatostoma pseudodon (Burrow, $1815)^{* 0}$; Phidiana lascrucensis Bertsch \& Ferreira, 1974a ; Polybranchia viridis (Deshayes, 1857)*a; Siphonaria maura Sowerby, 1835; Spurilla neapolitana (Delle Chiaje, 1841) ${ }^{\circ}$; Vasula melones (Duclos, 1832)*o; Vermetidae: (indet.*; indet. $\mathrm{A}^{\mathrm{o}}$; indet. $\mathrm{B}^{*}$; indet. $\mathrm{C}^{*}$; indet. $\mathrm{D}^{\mathrm{a}}$ ).

Chiton (Chiton) stokesii Broderip in Broderip \& Sowerby, 1832*; Polyplacophora: ( indet.*o; indet. $\mathrm{A}^{*}$ ).

Antillesoma antillarum (Grube, 1858) ${ }^{\text {oa }}$; Phascolosoma perlucens Baird, 1868*oa; Phascolosoma sp.*

Polychaeta indet. ; Amphinomidae indet.*; Cirratulidae indet. ; Eunicidae indet.*; Nereididae indet.*oa; Onuphidae indet.*; Phyllodocidae indet.*oa; Polynoidae indet.*; Sabellariidae: Phragmatopoma attenuata Hartman, 1944*o; Sabellidae: (indet. ${ }^{\circ}$; indet. $\mathrm{A}^{*}$; indet. $\mathrm{B}^{\mathrm{o}}$ ); Serpulidae: (indet.*; indet. $\mathrm{A}^{\mathrm{a}}$; indet. $\mathrm{B}^{\mathrm{o}}$ ); Syllidae indet. ${ }^{* 0}$; Terebellidae indet. ${ }^{* \text { oa }}$.

Alpheidae indet.*oa, Daira americana Stimpson, $1860^{\circ}$; Eriphia squamata Stimpson, 1860*0a, Pachygrapsus transversus (Gibbes, 1850)*oa; Pinnotheridae indet.*; Isopoda indet.*; Amphipoda indet.* 
CUADRO 2 (Continuación)

\begin{tabular}{|c|c|}
\hline Filo/Subfilo/Clase & Taxones \\
\hline Arthropoda/Crustacea/Maxillopoda (5) & $\begin{array}{l}\text { Chthamalus panamensis Pilsbry, } 1916^{* \mathrm{oa}} \text {; Megabalanus coccopoma (Darwin, } \\
\text { 1854)*; Microeuraphia rhizophorae (De Oliveira, 1940)*oa; Tetraclita stalactifera } \\
\left(\text { Lamarck, 1818)*o; Cirripedia indet. } \mathrm{A}^{* \mathrm{o}} \text {. }\right.\end{array}$ \\
\hline Echinodermata/Asteroidea (2) & Pharia pyramidata $(\text { Gray, } 1840)^{\circ}$; Phataria unifascialis $(\text { Gray, } 1840)^{\circ}$ \\
\hline Echinodermata/Echinoidea (3) & $\begin{array}{l}\text { Diadema mexicanum A. Agassiz, } 1863^{* 0} \text {; Echinometra vanbrunti A. Agassiz, } \\
\left.1863^{\text {oa }} \text {, Eucidaris thouarsii (L. Agassiz \& Desor, } 1846\right)^{\circ}\end{array}$ \\
\hline Echinodermata/Ophiuroidea (6) & $\begin{array}{l}\text { Ophiocoma: (O. aethiops Lütken, } 1859^{* \text { oa }} \text {; O. alexandri Lyman, } 1860^{* \text { oa }} \text { ); } \\
\text { Ophiothrichidae indet. }{ }^{\text {o }} \text { Ophiuroidea: (indet. }{ }^{* 0} \text {; indet. }{ }^{\text {o; indet. }} \mathrm{A}^{\mathrm{o}} \text { ) }\end{array}$ \\
\hline Echinodermata/Holothuroidea (4) & $\begin{array}{l}\text { Holothuroidea: (indet. }{ }^{\text {oa }} \text {; indet. A }{ }^{\mathrm{a}} \text { ); Holothuria inornata Semper, } 1868^{\mathrm{a}} \text {; } \\
\text { Holothuria } \text { sp.*a. }\end{array}$ \\
\hline Chordata/Urochordata/Ascidiacea (2) & Ascidiacea indet. ${ }^{\text {a }}$ Rhopalaea birkelandi Tokioka, $1971^{* 0 a}$. \\
\hline Chordata/Vertebrata/Actinoterygii (22) & 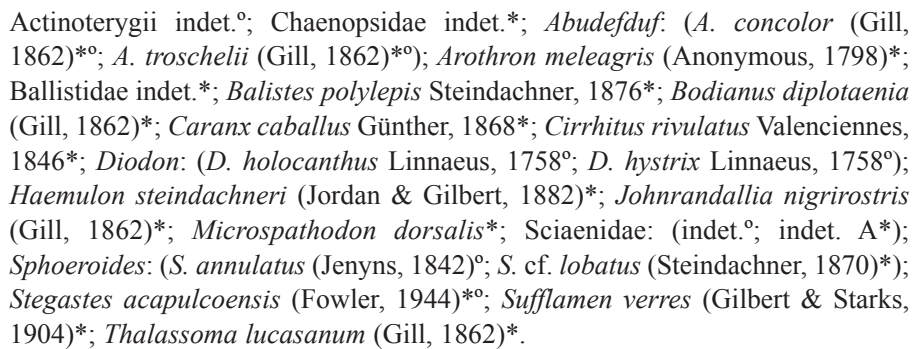 \\
\hline
\end{tabular}

\section{CUADRO 3}

Riqueza de taxones por grupo de organismos según zona de la costa rocosa de Bahía Panamá, Pacífico Norte. Agosto 2013. Se indica el valor de probabilidad de Chi cuadrado

\begin{tabular}{lccccc}
\multicolumn{1}{c}{ Taxones } & Total & Expuesto & Medio & Protegido & $\chi 2, \mathrm{p}$ \\
Autótrofos & 16 & 16 & 10 & 5 & 0.053 \\
Peces & 22 & 17 & 8 & 0 & $<0.001$ \\
Mollusca & 46 & 31 & 22 & 15 & $<0.001$ \\
Polychaeta & 17 & 11 & 10 & 4 & 0.143 \\
Crustacea & 12 & 11 & 8 & 5 & 0.328 \\
Echinodermata & 14 & 5 & 12 & 7 & 0.095 \\
Otros & 30 & 17 & 68 & 38 & 0.021 \\
Total Invertebrados & 119 & 75 & 87 & 43 & $<0.001$ \\
Total & 157 & 109 & & $<0.001$ \\
\hline
\end{tabular}

Sumando a lo anterior, en el Cuadro 4, se puede observar que 56 especies solo se encontraron en la zona expuesta y no en otras zonas, de modo contrario a la zona protegida donde solo 13 especies fueron propias de esa zona. El número de especies compartidas entre las zonas fue de 50 entre la parte externa y la media, y de solo 28 de estas dos zonas, con la protegida. Esto indica que la similitud fue baja entre zonas, siendo su menor valor entre la zona expuesta con la protegida, de apenas un $36 \%$ (Cuadro 4).

El patrón de cambio de la zona expuesta con mayor riqueza de taxones a la zona protegida con poca biota, se dio en estos microhábitats, salvo para la fauna que habitaba las grietas. Así mismo, en las grietas la riqueza tendió a ser menor que la determinada sobre 
CUADRO 4

Similitud entre las zonas de la costa rocosa según el índice de Sorensen (Números subrayados) basado en las listas de especies encontradas (incluidos los peces y autótrofos). La diagonal con números en negrita son las especies que solo se encontraron en cada zona. Los números en cursiva son las especies compartidas entre las zonas. Bahía Panamá, Costa Rica

\begin{tabular}{lccc}
\multicolumn{1}{r}{ Zona } & Expuesto & Medio & Protegido \\
Expuesto & $\mathbf{5 6}$ & 50 & 28 \\
Medio & $\underline{0.52}$ & $\mathbf{3 2}$ & 28 \\
Protegido & $\underline{0.36}$ & $\underline{0.43}$ & $\mathbf{1 3}$ \\
\hline
\end{tabular}

CUADRO 5

Riqueza de taxones por zona de exposición al oleaje, según microhábitat en las rocas de Bahía Panamá, Costa Rica

\begin{tabular}{lcccc}
\multicolumn{1}{c}{ Zona/microhábitat } & Dentro de grietas & Bajo la roca & Sobre la roca & Total \\
Expuesto & 11 & 18 & 48 & 77 \\
Medio & 11 & 18 & 40 & 69 \\
Protegido & 11 & 6 & 21 & 38 \\
\hline
\end{tabular}

las rocas (superficie expuesta); la riqueza de la biota que estaba habitando u ocultándose bajo las rocas fue el doble o hasta cuatro veces mayor que la de las grietas (Cuadro 5).

En adición a los patrones anteriores, también se encontró que la similitud de la composición de organismos en los tres microhábitats se redujo al comparar los extremos del gradiente de expuesto a protegido (Cuadro 6), lo que indica que la fauna cambia en identidad dependiendo de la zona de la costa donde habiten, respecto al oleaje (variación horizontal). Este patrón se cumplió para los tres microhábitats, pero la similitud de la fauna hallada bajo las rocas fue baja (30-20\%), mientras esa similitud aumenta a nivel medio (40-50\%) para la fauna sobre las rocas (Cuadro 6). Finalmente, la fauna que habitaba en las grietas fue la más similar entre las zonas, con valores de 55 a $73 \%$ (Cuadro 6).

\section{CUADRO 6}

Similitud (Sorensen) entre las zonas de exposición al oleaje, según micro-hábitat en las rocas de Bahía Panamá, Costa Rica

\begin{tabular}{lccc}
\multicolumn{1}{c}{ Zona/Microhabitat } & Bajo & Sobre & Grietas \\
Expuesto-Medio & 0.31 & 0.54 & 0.73 \\
Medio-Protegido & 0.18 & 0.45 & 0.64 \\
Expuesto-Protegido & 0.18 & 0.41 & 0.55 \\
\hline
\end{tabular}

\section{DISCUSIÓN}

Variación vertical de la comunidad: El patrón de zonación vertical se acerca bastante a la reportada por Fischer (1981) para la costa Pacífica de Costa Rica y la identidad de los organismos dominantes resultó muy similar a la de Bahía Salinas, Guanacaste, una localidad situada más al norte que Bahía Panamá (Sibaja-Cordero \& Cortés, 2008). El cambio en distribución de organismos desde la marea baja al supralitoral se debe a la desecación, la competencia por espacio y evitar la depredación, como ha sido ampliamente documentado a nivel mundial (Connell, 1972; Menge \& Sutherland, 1976; Garrity, 1984). Además, se encontró un patrón de variación similar al detectado por Sibaja-Cordero \& Vargas-Zamora (2006), que establecieron que la variación en composición de los organismos del intermareal es mayor en los estratos cercanos a la marea baja y dicha variación horizontal se va reduciendo en sentido vertical, siendo mínima en la zona supralitoral; esto se da porque las condiciones en los niveles inferiores son más favorables, albergando una mayor diversidad (Connell, 1972; Garrity, 1984), de modo contrario a los niveles superiores más expuestos, y por lo tanto menos diversos, poblados por 
pocas especies caracterizadas por presentar adaptaciones destinadas a soportar la desecación (Connell, 1972; Garrity, 1984; Lubchenco et al., 1984).

Variación horizontal de la comunidad: Se presenta asimismo en este estudio la variación horizontal en esta zona rocosa, pese a ser una pequeña bahía ubicada dentro de otra (Bahía Culebra), ubicada dentro del Golfo de Papagayo. Existe un patrón marcado de cambio desde la zona más expuesta hacia la más protegida del oleaje y corrientes. Este cambio no solo se dio para las especies dominantes sobre la roca, en su cobertura y abundancia, sino que se reflejó también en los organismos que habitan o se refugian bajo las rocas. La identidad de la fauna (presencia o no de ciertas especies), también mostró una diferenciación de leve a marcada siguiendo el mismo gradiente de expuesto a protegido, en este caso incluso para los habitantes de las grietas. El cambio en la identidad puede ser explicado en parte en función de lo sugerido por Archambault \& Bourget (1996), donde la variación horizontal a pequeña escala $(\mathrm{cm})$ de la heterogeneidad espacial hace variar la cobertura y abundancia, pero para ver un cambio en riqueza de especies se necesita ver la heterogeneidad del hábitat en una escala de metros.

Igualmente, se detectó un patrón similar en el cambio de especies, entre la parte interna y la externa como en el Golfo de Nicoya (SibajaCordero \& Vargas-Zamora 2006). Araújo et al. (2005) indican que la variación horizontal en los ensambles de especies está relacionada con cambios en exposición al oleaje y topografía del intermareal, e indican que las interacciones entre especies pueden provocar distribución en tipo mosaico. Así, patrones de cambio como el hallado en el presente estudio, i.e. desde zonas protegidas a expuestas, se mantienen por el efecto de las corrientes y oleaje, pero varían en la composición de la cobertura y abundancia. Smith \& Simpson (2002) indican que la exposición al oleaje es un factor de cambio incluso en una escala de metros o decenas de metros, pero factores que actúan a una escala menor pueden modificar bastante el patrón esperado.

Se notó que la playa de arena situada en la parte interna de esta bahía contribuye con sedimento que se deposita sobre la zona rocosa, lo que no ocurre en la plataforma de la zona más expuesta, donde la bahía es más profunda (2-3 m) y hay menos deposición de sedimentos sobre las rocas del intermareal. El cambio es menor entre sitios aledaños y mayor entre los extremos aquí evaluados, lo que coincide con los resultados de Davidson (2005) para otro litoral rocoso, donde indica que el gradiente horizontal de la costa cambia la composición dentro de cada estrato vertical. Esto indicaría que se puede hacer cierta extrapolación de este patrón en otros accidentes geográficos similares en la región.

El que la similitud de la fauna que habita en las grietas de la zona intermareal haya sido mayor que la de organismos de la epibiota en las rocas, y aún mayor que la de los organismos bajo las rocas, se debe a que son menos las especies adaptadas para la vida dentro de grietas. Pepe (1985) encontró en Playas del Coco (al sur de Bahía Panamá) solo nueve especies; no obstante en el presente estudio, debajo de las rocas habitaban muchos organismos sésiles, así como numerosas especies móviles que se refugian ahí durante el día o la marea baja, para escapar de la desecación o la depredación. Algunos de estos organismos salen a forrajear o interactuar por las noches o en marea alta, por lo cual esto contribuye en generar una fauna más heterogénea. Hay especies que se pueden encontrar a lo largo de la costa, pero otras solo tienden a darse en ciertas secciones del intermareal, dependiendo del grado de exposición.

Finalmente, la cantidad de peces avistada en el infralitoral y las pozas intermareales, muchos de ellos forrajeando algas o invertebrados, indica que estas zonas son de gran importancia para el mantenimiento de poblaciones de estos organismos, como indica Ortega (1986). Resultados de variación espacial en el forrajeo de peces se ha reportado para Panamá por Garrity et al. (1986), quienes indican que hay gran variación entre sitios. Lubchenco et al. (1984) indican que 
la depredación puede reducirse con el aumento en la turbidez, pero Ortega (1986) y Garrity et al. (1986) indican que no tiene un efecto y que el mayor impedimento es el incremento en la fuerza del oleaje. En el presente estudio, los peces avistados muestran una menor abundancia hacia el interior de la bahía, donde la profundidad es mucho menor y hay más turbidez, pero también la cobertura y cantidad de posibles presas es menor. Además, al estar dentro de otra bahía, esta costa es más protegida que zonas expuestas como las mencionadas por Ortega (1986) y Garrity et al. (1986).

Variación temporal de la comunidad: Otras causas de cambio en los intermareales se deben a competencia entre las especies, establecimiento o no de reclutas, herbivoría o efecto de los depredadores; al igual que en otros estudios en zonas templadas y tropicales, estas causas se han vinculado a las variaciones en la columna del agua y de corrientes como desencadenantes del cambio en estos procesos (Sutherland \& Ortega, 1986; Menge et al., 1997, 2003). La costa Pacífica más al norte de Costa Rica está influenciada por el afloramiento estacional, debido al efecto de vientos alisios sobre las masas de agua durante la estación seca de diciembre a abril (Alfaro et al., 2012), presentado aguas más frías y con más nutrientes que hacen variar la biota de los intermareales rocosos (Sibaja-Cordero \& Cortés, 2008). Por el contrario, en la estación lluviosa, donde no hay afloramiento, el sistema se hace menos productivo. En ese sentido, parte de los cambios observados entre 2007 y 2013 en Bahía Panamá, pueden deberse a dicha estacionalidad, donde las especies pueden reducir o incrementar su cobertura y abundancia de acuerdo con cambios en los factores ambientales. En ese sentido, algas incrustantes como Ralfsia sp. se incrementaron en 2013, mientras algas rojas frondosas y verdes tendieron a reducirse (por la menor presencia de nutrientes en el agua), salvo C. sertularoides (un alga invasiva, FernándezGarcía et al., 2012) que aumentó en el interior de la bahía. Pese a que las formas incrustantes en algas tienden a dominar más cuando hay una mayor intensidad de la herbivoría (Hawkins \&
Hartnoll, 1983; Little \& Kitching, 1996), en nuestro caso muchos gasterópodos herbívoros presentaron una tendencia a la reducción en sus poblaciones en 2013, en el infralitoral. Estos cambios en la composición de herbívoros desembocan en variaciones en los ensambles de otras especies del intermareal (Menge \& Sutherland, 1976; Anderson \& Underwood, 1997).

Otra evidencia en el sentido del cambio en la biota debido a que las aguas son menos productivas, fue lo observado en el muestreo de 2013, cuando se presentó una reducción de cobertura en los organismos suspensívoros como, el bivalvo Brachidontes sp., cirripedios y anémonas; la única excepción fue el bivalvo S. palmula, que incrementó su cobertura. Cambios similares han sido hallados en California, EEUU, dependiendo de la localidad y la intensidad del afloramiento (Connolly \& Roughgarden, 1999). La estimación de cambio temporal está graficada basada en los centroides del análisis multivariable, pero la prueba se hizo por medio de permutaciones con todas las cuadrículas, para tomar en cuenta la variabilidad espacial de 2007; sin embargo, se recomienda en el futuro realizar un monitoreo con igual número de réplicas, siguiendo los métodos recogidos en Underwood \& Chapman (1998), para corroborar la evidencia aquí presentada.

Biodiversidad: Tradicionalmente se ha categorizado a los intermareales rocosos tropicales como poco diversos en comparación con localidades de clima templado, o bien con otros hábitats marinos tropicales (Paine, 1966; ver Tabla 3 en Davidson, 2005); sin embargo, los datos aquí presentados indican que los invertebrados y algas que no forman grandes franjas, parches o agregaciones aportan un gran porcentaje de la biodiversidad observada. Por lo general, estos se encuentran en los microhábitats más crípticos del intermareal y poblando las pozas intermareales (Davidson, 2005). Así, en función de los organismos asociados a la zona rocosa de Bahía Panamá, se deduce que estos ambientes costeros de substrato duro contienen una alta biodiversidad en los trópicos. Las causas son la heterogeneidad 
de microhábitats y la presencia de diversos gradientes antes mencionados.

Conclusiones: La composición de la fauna de la zona rocosa cambia siguiendo un gradiente vertical, de acuerdo a los niveles de marea, como ha sido referido previamente para estos ambientes; además, esta muestra un descenso en la variación de la composición desde el infralitoral al supralitoral. Así mismo, se encontró un cambio de la composición y la fauna, siguiendo un gradiente desde las zonas protegidas a las más expuestas. Este patrón de cambio horizontal en la costa se mantiene temporalmente, pero en cada fecha de muestreo se encontró que los ensambles de especies no coincidían en su totalidad. Este grado de cambio podría estar asociado a variaciones en las características del medio, según la influencia o no del afloramiento estacional. Los componentes de la biota están repartidos entre los microhábitats del intermareal, siendo los principales formadores del paisaje las formas epibentónicas, mientras la fauna críptica (bajo rocas y en grietas) parecen ser los mayores contribuyentes a la biodiversidad total. Un monitoreo de la costa es necesario para generar modelos ecológicos que sirvan para permitir formular predicciones en este tipo de accidentes geográficos costeros, y debería tomarse en cuenta los niveles de variación espacial y temporal, para, en definitiva, mejorar la conservación de estos medios en el trópico.

\section{AGRADECIMIENTOS}

Se agradece a Jorge Cortés, y al proyecto \#808-96-298 de la Vicerrectoría de Investigación de la Universidad de Costa Rica, por el transporte para realizar el muestreo de Diciembre de 2007.

\section{REFERENCIAS}

Alfaro, E. J., Cortés, J., Alvarado, J. J., Jiménez, C., León, A., Sánchez-Noguera, C., Nivia-Ruiz, J. \& RuizCampos, E. (2012). Clima y variabilidad climática de la temperatura subsuperfical del mar en Bahía
Culebra, Guanacaste, Costa Rica. Revista de Biología Tropical, 60 (Suplemento 2), 159-171.

Anderson, M. J. \& Underwood A. J. (1997). Effects of gastropod grazers on recruitment and succession of an estuarine assemblage: a multivariate and univariate approach. Oecologia, 109, 442-453.

Araújo, R., Bárbara, I., Sousa-Pinto, I. \& Quintino, V. (2005). Spatial variability of intertidal rocky shore assemblages in the northwest coast of Portugal. Estuarine, Coastal and Shelf Science, 64, 658-670.

Archambault, P. \& Bourget, E. (1996). Scales of coastal heterogeneity and benthic intertidal species richness. Marine Ecology Progress Series, 136, 111-121.

Bakus, G. J. (1968). Zonation in marine gastropods of Costa Rica and species diversity. Veliger, 10, 207-211.

Clarke, K. R. \& Warwick, R. M. (1994). Change in Marine Communities: An Approach to Statistical Analysis and Interpretation. Plymouth: Bourne, Bournemouth.

Connell, J. H. (1972). Community interactions on marine rocky intertidal shores. Annual Review of Ecology and Systematics, 3, 169-192.

Connolly, S. R. \& Roughgarden, J. (1999). Theory of marine communities: competition, predation, and recruit-ment-dependent interaction strength. Ecological Monographs, 69, 277-296.

Davidson, I. C. (2005). Structural gradients in an intertidal hard-bottom community: examining vertical, horizontal, and taxonomic clines in zoobenthic biodiversity. Marine Biology, 146, 827-839.

Dean, H. K. (2001). Marine Biodiversity of Costa Rica: The phyla Sipuncula and Echiura. Revista de Biología Tropical, 49 (Suplemento 2), 85-90.

Fernández-García, C., Cortés, J., Alvarado, J. J. \& NiviaRuiz, J. (2012). Physical factors contributing to the benthic dominance of the alga Caulerpa sertularioides (Caulerpaceae, Chlorophyta) in the upwelling Bahía Culebra, north Pacific of Costa Rica. Revista de Biología Tropical, 60 (Suplemento 2), 93-107.

Fischer, R (1981). Bioerosion of basalt of the Pacific coast of Costa Rica. Senckenbergiana maritima, 13, 1-41.

Garrity, S. D. (1984). Some adaptations of gastropods to physical stress on a tropical rocky shore. Ecology, $65,559-574$.

Garrity, S. D., Levings, S. C. \& Caffey, H. M. (1986). Spatial and temporal variation in shell crushing by fishes on rocky shores of Pacific Panama. Journal of Experimental Marine Biology and Ecology, 103, 131-142.

Hammer, Ø., Harper, D. A. T. \& Ryan, P. D. (2001). Past: Paleontological Statistics Software Package for Education and Data Analysis. Palaeontologia Electronica, 4, 1-9. 
Hawkins, S. J. \& Hartnoll, R. G. (1983). Grazing of intertidal algae by marine invertebrates. Oceanography and Marine Biology Annual Review, 21, 195-282.

Humann, P. \& DeLoach, N. 2004. Reef Fish Identification: Baja to Panama. Jacksonville, Florida: New World Publications.

Keen, A. M. (1971). Sea Shells of Tropical West America. Marine Mollusks from Baja California to Peru, (2nd ed). California: Stanford University Press.

Laguna, J. E. (1985). Systematics, Ecology and Distribution of Barnacles (Cirripedia; Thoracica) of Panama, Including an Analysis of Provincialism in the Tropical Eastern Pacific. M.Sc. Thesis. San Diego, California: University of California.

Little, C. \& J. A. Kitching. (1996). The Biology of Rocky Shores. Oxford: Oxford University Press.

Lubchenco, J., Menge, B. A., Garrity, S. D., Lubchenco, P., Gaines, S. D., Ashkenas, L. R., Emlet, R., Lucas, J. \& Strauss, S. (1984). Structure, persistence and role of consumers in a tropical rocky intertidal community (Taboguilla Island, Bay of Panama). Journal of Experimental Marine Biology and Ecology, 78, 23-73.

Menge, B. A, Daley, B. A, Wheeler, P. A, Dahlhoff, E., Sanford, E. \& Strub, P. T. (1997). Benthic-pelagic links and rocky intertidal communities: bottom-up effects on top-down control? Proceedings of the National Academy of Sciences of the United States of America, 94, 14530-14535.

Menge, B. A. \& Lubchenco, J. (1981). Community organization in temperate and tropical rocky intertidal habitats: prey refuges in relation to consumer pressure gradients. Ecological Monographs, 51, 429-450.

Menge, B. A., Lubchenco, J., Bracken, M. E. S., Chan, F., Foley, M. M., Freidenburg, T. L., Gaines, S. D., Hudson, G., Krenz, C., Leslie, H., Menge, D. N. L., Russell, R. \& Webster, M. S. (2003). Coastal oceanography sets the pace of rocky intertidal community dynamics. Proceedings of the National Academy of Sciences of the United States of America, 100, 12229-12234.

Menge, B. A. \& Sutherland, J. P. (1976). Species diversity gradients: synthesis of the roles of predation, competition, and temporal heterogeneity. American Naturalist, 110, 351-369.

Miller, A. C. (1974). A Comparison of Gastropod Diversity and Trophic Structure in the Rocky Intertidal Zone of the Temperate and Tropical West America. Ph.D. Thesis. Eugene, Oregon: University of Oregon.

Ortega, S. (1986), Fish predation on gastropods on the Pacific coast of Costa Rica. Journal of Experimental Marine Biology and Ecology, 97, 181-191.
Ortega, S. (1987). Habitat segregation and temporal variation in some tropical intertidal populations. Journal of Experimental Marine Biology and Ecology, 113, 247-265.

Paine, R. T. (1966). Food web complexity and species diversity. American Naturalist, 100, 66-75.

Pepe, P. J. (1985). Littoral endolithic fauna of the Central American Isthmus. Revista de Biología Tropical, 33, 191-194.

Quinn, G. P. \& Keough, M. J. (2003). Experimental Design and Data Analysis for Biologists. Cambridge: Cambridge University Press.

Sibaja-Cordero, J. A. (2008). Vertical zonation in the rocky intertidal at Cocos Island (Isla del Coco), Costa Rica: A comparison with other tropical locations. Revista de Biología Tropical, 56 (Suplemento 2), 171-187.

Sibaja-Cordero, J. A. \& Cortés, J. (2008). Vertical zonation of rocky intertidal organisms in a seasonal upwelling area (Eastern Tropical Pacific). Revista de Biología Tropical, 56 (Suplemento 4), 91-104.

Sibaja-Cordero, J. A., \& Vargas-Zamora, J.A. (2006). The vertical zonation of epifauna and algae species in rocky substrates of the Gulf of Nicoya, Costa Rica. Revista de Biología Tropical, 54 (Suplemento 1), 49-67.

Smith, S. D. A. \& Simpson, R. D. (2002). Spatial variation in the community structure of intertidal habitats at Macquarie Island (sub-Antarctic). Antarctic Science, 14, 374-384.

Spight, T. M. (1977). Diversity of shallow-water gastropod communities on temperate and tropical beaches. American Naturalist, 111, 1077-1097.

Sutherland, J. P. \& Ortega, S. (1986). Competition conditional on recruitment and temporary escape from predators on tropical rocky shore. Journal of Experimental Marine Biology and Ecology, 95,155-166.

Underwood, A. J. \& Chapman, M. G. (1998). A method for analysing spatial scales of variation in composition of assemblages. Oecologia, 117, 570-578.

Villalobos, C. R. (1980a). Variations in population structure in the genus Tetraclita (Crustacea, Cirripedia) between temperate and tropical populations. III. T. stalactifera in Costa Rica. Revista de Biología Tropical, 28, 193-201.

Villalobos, C. R. (1980b). Algunas consideraciones sobre el efecto de los factores físicos y biológicos en la estructura de una comunidad de algas en el Pacífico de Costa Rica. Brenesia, 18, 289-300.

Willis, S. \& Cortés, J. (2001). Mollusks of Manuel Antonio National Park, Pacific Costa Rica. Revista de Biología Tropical, 49 (Suplemento 2), 25-36. 
